\title{
Differences in the expression of five senescence markers in oral cancer, oral leukoplakia and control samples in humans
}

\author{
ANTONIO BASCONES-MARTÍNEZ ${ }^{1}$, MERCEDES LÓPEZ-DURÁN ${ }^{1}$, JORGE CANO-SÁNCHEZ ${ }^{1}$, \\ LYDIA SÁNCHEZ-VERDE ${ }^{2}$, ANA DÍEZ-RODRÍGUEZ ${ }^{2}$, PABLO AGUIRRE-ECHEBARRÍA ${ }^{3}$, \\ EMILIO ÁLVAREZ-FERNÁNDEZ ${ }^{3}$, MIGUEL ANGEL GONZÁLEZ-MOLES ${ }^{4}$, JAIME BASCONES-ILUNDAIN ${ }^{1}$, \\ LORENZO LO MUZIO ${ }^{5,6}$ and JULIÁN CAMPO-TRAPERO ${ }^{1}$ \\ ${ }^{1}$ Department of Oral Surgery, Oral Medicine and Periodontology, Dental School, Complutense University of Madrid; \\ ${ }^{2}$ Inmunohistochemistry and Histology Unit, CNIO (Spanish National Cancer Centre); ${ }^{3}$ Department of Pathology, \\ Hospital Gregorio Marañón, Madrid; ${ }^{4}$ Department of Oral Medicine, Dental School, Granada University, \\ Granada, Spain; ${ }^{5}$ Department of Surgical Sciences, University of Foggia, Foggia; \\ ${ }^{6}$ IRCCS CROB - National Cancer Insititute of Oncology of Basilicata, Rionero in Vulture, Potenza, Italy
}

Received December 11, 2011; Accepted January 30, 2012

DOI: 10.3892/ol.2012.649

\begin{abstract}
Oncogene-induced senescence (OIS) may be a response to oncogenic activation, acting as a natural barrier against carcinogenesis at a premalignant stage. Thus, numerous cells in premalignant lesions enter senescence, but none or few in malignant tumours. This event could be due to the loss of senescence pathway effectors, including p16 (INK4a)-pRb or ARF-p53. The aim of this study was to characterize and compare the expression of certain senescent markers between oral precancer and cancer tissue samples. The expression of cyclin D1, Rb, maspin, p53 and mouse double minute 2 (MDM2) was analyzed in 20 paraffin-embedded tissue samples of normal oral mucosa (NOM), 14 samples of oral leukoplakia without dysplasia (OLD-), 11 samples of leukoplakia with dysplasia (OLD+) and 15 samples of oral squamous cell carcinoma (OSCC) by immunohistochemistry in tissue arrays. The expression of p16-pRb pathway markers, cyclin D1, maspin and $\mathrm{Rb}$, was more frequent in OLD+ samples than in OSCC samples, although a statistical significance was only observed for maspin $(\mathrm{P}=0.036)$. Cyclin $\mathrm{D} 1$ expression was also significantly more frequent in OLD- samples vs. NOM samples. For the ARF-p53 pathway, the expression of p53 and MDM2 was significantly more frequent in the OLD- samples compared to in the NOM ones. These findings may indicate a role for cellular senescence in oral carcinogenesis, considering maspin
\end{abstract}

Correspondence to: Ms. Mercedes Lopez-Duran, Department of Oral Surgery, Oral Medicine and Periodontology, Dental School, Complutense University of Madrid, Plaza de Ramón y Cajal, 3. Ciudad Universitaria, Madrid 28040, Spain

E-mail: lopezduran.m@gmail.com

Key words: senescence, oral cancer, oral leukoplakia, cyclin D1, maspin, Rb, p53, MDM2 as a reliable senescence marker and prognostic factor in oral premalignant lesions.

\section{Introduction}

Cellular senescence was first reported in cultured human fibroblasts (1). Hayflick and Moorhead observed permanent replication arrest in phase G1 of the cell cycle following a certain number of replications, but without loss of viability or metabolic capacity (1). This physiological limit is thought to be a mechanism for avoiding cell immortality or preventing prolongation of the replicative life of cells, which would increase their susceptibility to gain successive mutations, and thus favour their progression to malignancy (2). It was recently demonstrated that this suppression of the proliferative capacity of cells may also be triggered by oncogene-induced senescence (OIS) (2). Various studies of premalignant lesions have proposed a role for OIS as an anti-oncogenic response in vivo, describing senescent cells in premalignant lesions, but not in tumours, and concluding that OIS may act as a barrier against malignant transformation. Accordingly, only cells that lose this senescent response are transformed from a benign state (premalignant lesion) to malignancy (carcinoma). However, given the complexity of the carcinogenic process, this does not appear to be a cause-effect correlation, but rather a further expression of carcinogenesis $(2,3)$.

Three pathways have been reported to induce senescence: the ARF/p53/p21 pathway, also involved in apoptosis; the p16/ $\mathrm{Rb} / \mathrm{E} 2 \mathrm{~F}$ pathway, more specifically related to senescence; and a third, less well-documented pathway via retinoic acid receptors (4). The expression of molecular markers of these pathways in different types of carcinomas have been studied and correlated with prognosis. To test the hypothesis that OIS acts as an anti-carcinogenic barrier in the premalignant stage, we compared the expression of these markers between premalignant and malignant oral lesions. The potential value of findings would be to offer a basis for predicting the malignant 
transformation of a premalignant lesion (e.g., leukoplakia or erythroplakia), and to identify senescence markers that may serve as therapeutic targets, using senescence as a physiological anti-tumour mechanism, by increasing their expression in premalignant oral lesions.

Thus, the objectives of this study were to characterize the expression of various markers involved in the main senescence pathways in oral leukoplakia lesions, and to compare this expression with that of the markers in oral squamous cell carcinomas (OSCCs). We selected markers studied in other types of carcinomas for which, however, little data are available at the oral level, despite the fact that worldwide over half of the 500,000 new cases of head and neck cancer per year are in the oral cavity, with a five-year survival rate of only $55 \%$ (5). Immunohistochemistry was performed on tissue microarray (TMA) sections to study cyclin D1, maspin and $\mathrm{Rb}$ for the specific senescence pathway p16-pRb; and p53 and mouse double minute 2 (MDM2) for the apoptosis/senescence ARF-p53 pathway.

\section{Materials and methods}

Study subjects. TMA analysis was conducted in 20 samples of normal oral mucosa (NOM), 14 of leukoplakia without dysplasia (OLD-), 11 of leukoplakia with dysplasia (OLD+) and 15 of OSCC. Paraffin-embedded samples were provided by the Gregorio Marañon Hospital in Madrid, the School of Dentistry of Madrid Complutense University and the Department of Surgical Sciences of the University of Foggia. Data were gathered from patient clinical records with respect to their age, gender, medication, systemic disease, tobacco use, personal history of oral cancer and on the site from which the sample derived. Information was also collected on the degree of dysplasia (mild/moderate/severe) in cases of OLD+, and on the clinical/histological type and TNM classification in OSCC cases (International Union Against Cancer criteria).

Data confidentiality was maintained by replacing all patient identifiers with numerical codes. Informed consent was obtained from all participating patients, and the use of biopsied tissue was approved by the Ethics Committees of the two institutions.

TMA construction. TMA was constructed from the anonymous samples at the Spanish National Cancer Research Centre. The orientation of the sample in the paraffin block was determined by hematoxylin-eosin staining. A specialist in oral pathology identified the most characteristic epithelial areas of oral dysplasia or carcinoma based on optical microscope observations. The same pathologist labelled two representative areas of each sample for the extraction of two cylinders for the TMA. Labelling was conducted in paraffin blocks and hematoxylin and eosin-stained slides. The pathologist was blinded to the clinical data of the corresponding patients.

The TMA was then constructed. The array receptors were paraffin blocks that were previously smoothed to ensure that cylinders had the same angle of entry. Samples were introduced in duplicate into a single TMA, and were ordered following a random number program to minimize immunostaining bias. The TMA was prepared with 120 cylinders corresponding to
60 patients. Samples of pancreas, tonsil, kidney and lung were also added in duplicate as internal controls, and an additional tonsil sample was included for the TMA orientation. The construction was conducted using a manual TMA apparatus (Beecher Instruments, Sun Prairie, WI, USA). Each cylinder had a diameter of $1 \mathrm{~mm}$ and was placed, using digital micrometry, $1.5 \mathrm{~mm}$ from its neighbors. Following completion of the cylinder transfer, the TMA was incubated for $6 \mathrm{~min}$ at $64^{\circ} \mathrm{C}$ to facilitate fusion of the cylinders with the paraffin. They were subsequently cooled and cut to a thickness of 3 microns. One section was stained with hematoxylin/eosin, and the remainder underwent immunohistochemical analysis for each study protein.

Immunohistochemical analysis. The immunoreaction of all study proteins was analysed. First, samples were deparaffinised and hydrated. A HIER (Heat-Induced Epitope Retrieval) stage was conducted in a sodium citrate buffer solution with $0.01 \mathrm{M}$ trisodium citrate solution or $1 \mathrm{mM}$ TE (Tris-EDTA) at $\mathrm{pH} 8.0$. It was then heated for $20 \mathrm{~min}$ at $98^{\circ} \mathrm{C}$ in a PT Link (Dako, Carpenteria, CA, USA) oven. Following heating, sections were rinsed in cold running water for $5 \mathrm{~min}$, then passed through Tris-buffered saline (TBS) at $\mathrm{pH}$ 7.4. The sections were then incubated with the corresponding antibodies, i.e., SP4 for cyclin D1 (NeoMarkers Fremont, CA, USA), polyclonal rabbit for maspin (Santa Cruz Biotechnology, Santa Cruz, CA, USA), G3-245BD for Rb (BD PharMingen, San Diego, CA, USA), DO-7 for p53 (Novocastra, Burlingame, CA, USA), IF2 for MDM2 (Calbiochem, Darmstadt, Germany) and MIB 1 for Ki-67 (Dako, Glostrup, Denmark). Following incubation with the primary antibodies, EnVision-FLEX (Dako) was used in Dako Autostainer Plus Link automatic equipment for the immunodetection of all markers with the exception of maspin, using diaminobenzidine chromogen as the substrate. Maspin was visualized by using the Leica Vision Bio System with a Leica BOND MAX immunostainer. Maspin antigen retrieval was performed with EDTA, using the same immunostainer. For the controls, incubations were performed with omission of the specific antibodies and with the inclusion of unrelated antibodies.

TMA analysis. Cylinder sections with no epithelium or containing $<50 \%$ of the original tissue were considered as non-representative samples and excluded from the immunostaining analysis. The degree of staining was determined in all cylinders remaining intact following the immunohistochemistry by an optical microscopy study (magnification, x10 and $\mathrm{x} 100$ ) of each cylinder. The staining was conducted by two members of the team, blinded at all times to the clinical data corresponding to the samples. A sample was only excluded when both cylinders were non-assessable.

Statistical analysis. Stat View v.5.0.1 (SAS Institute, Inc., NC, USA) and Statgraphics Plus v. 5.1 (Statistical Graphics Corp., Oswego, NY, USA) were used for the statistical analyses. The Chi-square test and Fisher's exact test (if $\mathrm{N}<5$ ) were used for the the comparison of staining among clinical stages and to establish differences in the studied proteins among the study groups (NOM, OLD-, OLD+ and OSCC). P $<0.05$ was considered to indicate a statistically significant difference. 
Table I. Clinicopathological characteristics of the patients by study group (NOM, OLD-, OLD+ and OSCC).

\begin{tabular}{|c|c|c|c|c|c|c|c|c|}
\hline \multirow[b]{2}{*}{ Variable } & \multicolumn{2}{|l|}{ NOM } & \multicolumn{2}{|c|}{ OLD- } & \multicolumn{2}{|c|}{ OLD+ } & \multicolumn{2}{|c|}{ OSCC } \\
\hline & No. & $\%$ & No. & $\%$ & No. & $\%$ & No. & $\%$ \\
\hline \multicolumn{9}{|l|}{ Age } \\
\hline Mean (years) & $50.6(15-80)$ & & 55.7 (26-76) & & $67.9(42-86)$ & & $67.8(48-87)$ & \\
\hline \multicolumn{9}{|l|}{ Gender } \\
\hline Male & 12 & 60 & 8 & 57.1 & 4 & 36.4 & 10 & 66.7 \\
\hline Female & 8 & 40 & 6 & 42.9 & 7 & 63.6 & 5 & 33.3 \\
\hline \multicolumn{9}{|l|}{ Tobacco } \\
\hline Yes & 7 & 35 & 10 & 71.4 & 6 & 54.6 & 13 & 86.7 \\
\hline No & 13 & 65 & 4 & 28.6 & 5 & 45.4 & 2 & 13.3 \\
\hline \multicolumn{9}{|l|}{ Alcohol } \\
\hline Yes & 4 & 20 & 5 & 35.7 & 3 & 27.3 & 9 & 60 \\
\hline No & 16 & 80 & 9 & 64.3 & 8 & 72.7 & 6 & 40 \\
\hline \multicolumn{9}{|l|}{ Localization } \\
\hline Gingiva & 20 & 100 & 2 & 14.3 & 0 & 0 & 2 & 13.3 \\
\hline Tongue and floor & 0 & 0 & 11 & 78.6 & 5 & 45.5 & 8 & 53.3 \\
\hline Other & 0 & 0 & 1 & 7.1 & 6 & 54.5 & 5 & 33.4 \\
\hline \multicolumn{9}{|l|}{ Dysplasia } \\
\hline Mild & - & - & - & - & 6 & 54.5 & - & - \\
\hline Moderate & - & - & - & - & 1 & 9.1 & - & - \\
\hline Severe & - & - & - & - & 4 & 36.4 & - & - \\
\hline \multicolumn{9}{|l|}{ T classification } \\
\hline $\mathrm{T} 1$ & - & - & - & - & - & - & 7 & 46.6 \\
\hline $\mathrm{T} 2$ & - & - & - & - & - & - & 6 & 40 \\
\hline $\mathrm{T} 3$ & - & - & - & - & - & - & 1 & 6.7 \\
\hline T4 & - & - & - & - & - & - & 1 & 6.7 \\
\hline \multicolumn{9}{|l|}{$\mathrm{N}$ classification } \\
\hline No & - & - & - & - & - & - & 9 & 60 \\
\hline N1 & - & - & - & - & - & - & 2 & 13.3 \\
\hline $\mathrm{N} 2$ & - & - & - & - & - & - & 4 & 26.7 \\
\hline N3 & - & - & - & - & - & - & 0 & 0 \\
\hline \multicolumn{9}{|l|}{ Clinical stage } \\
\hline I & - & - & - & - & - & - & 5 & 33.33 \\
\hline II & - & - & - & - & - & - & 2 & 13.33 \\
\hline III & - & - & - & - & - & - & 3 & 20 \\
\hline IV & - & - & - & - & - & - & 5 & 33.33 \\
\hline \multicolumn{9}{|l|}{ Histological type } \\
\hline G1 & - & - & - & - & - & - & 3 & 20 \\
\hline G2 & - & - & - & - & - & - & 8 & 53.3 \\
\hline G3 & - & - & - & - & - & - & 4 & 26.7 \\
\hline
\end{tabular}

NOM, normal oral mucosa; OLD+, leukoplakia with dysplasia, OLD-, leukoplakia without dysplasia; OSCC, oral squamous cell carcinoma.

\section{Results}

Clinicopathological characteristics. Table I shows the clinicopathological characteristics of the patients. No significance was observed in patient characteristics, such as age, gender, smoking status and alcohol consumption among the four study groups.
p16-pRb pathway. Fig. 1 shows examples of the expression of the markers (cyclin D1, maspin and Rb) of the senescence-specific pathway for each study group (NOM, OLD-, OLD+ and OSCC). Table II shows the immunohistochemistry results, expressed as positively or negatively stained samples. Statistical comparisons were performed between the following groups: NOM vs. OLD-; OLD vs. OLD+; OLD+ vs. OSCC and NOM vs. OSCC. 
Table II. Expression of p16-pRB senescence pathway markers.

\begin{tabular}{|c|c|c|c|c|c|c|c|c|c|}
\hline \multirow{2}{*}{$\begin{array}{l}\text { Type of } \\
\text { lesion }\end{array}$} & \multicolumn{3}{|c|}{ Cyclin D1 } & \multicolumn{3}{|c|}{ Maspin } & \multicolumn{3}{|c|}{$\mathrm{Rb}$} \\
\hline & Positive & Negative & P-value & Positive & Negative & P-value & Positive & Negative & P-value \\
\hline $\begin{array}{l}\text { NOM } \\
(\mathrm{n}=20)\end{array}$ & $0(0 \%)$ & $20(100 \%)$ & $0.021^{\mathrm{a}}$ & $19(95 \%)$ & $1(5 \%)$ & $1.000^{\mathrm{a}}$ & $13(65 \%)$ & $6(30 \%)$ & $0.102^{\mathrm{a}}$ \\
\hline $\begin{array}{l}\text { OLD- } \\
(\mathrm{n}=14)\end{array}$ & $4(28.5 \%)$ & $9(64.3 \%)$ & $0.241^{\mathrm{b}}$ & $14(100 \%)$ & $0(0 \%)$ & $0.440^{\mathrm{b}}$ & $13(92.8 \%)$ & $1(7.1 \%)$ & $1.000^{\mathrm{b}}$ \\
\hline $\begin{array}{l}\text { OLD+ } \\
(\mathrm{n}=11)\end{array}$ & $6(54.5 \%)$ & $4(36.3 \%)$ & $0.697^{c}$ & $10(91 \%)$ & $0(0 \%)$ & $0.036^{c}$ & $10(90.9 \%)$ & $0(0 \%)$ & $0.197^{c}$ \\
\hline $\begin{array}{l}\text { OSCC } \\
(\mathrm{n}=15)\end{array}$ & $7(46.6 \%)$ & $8(53.3 \%)$ & $<0.001^{d}$ & $7(46.6 \%)$ & $8(53.3 \%)$ & $0.002^{\mathrm{d}}$ & $10(66.6 \%)$ & $5(33.3 \%)$ & $0.919^{\mathrm{d}}$ \\
\hline
\end{tabular}

P-values indicate differences in proportions: ${ }^{a} \mathrm{NOM}$ vs. OLD-; ${ }^{\mathrm{b} O L D}-\mathrm{vs.} \mathrm{OLD+;}{ }^{\mathrm{O}} \mathrm{OLD}+\mathrm{vs}$. OSCC; ${ }^{\mathrm{d} N O M}$ vs. OSCC. Statistically significant differences are in bold. For cyclin D1, staining of more than the basal cell layer is considered positive. One sample was excluded (as non-valuable) from the cyclin D1 staining (1 OLD+); one from the maspin (1 OLD+); and two from the Rb staining (1 NOM and 1 OLD+). The same OLD+ sample (LC43) was excluded from all staining procedures. NOM, normal oral mucosa; OLD+, leukoplakia with dysplasia; OLD-, leukoplakia without dysplasia; OSCC, oral squamous cell carcinoma.

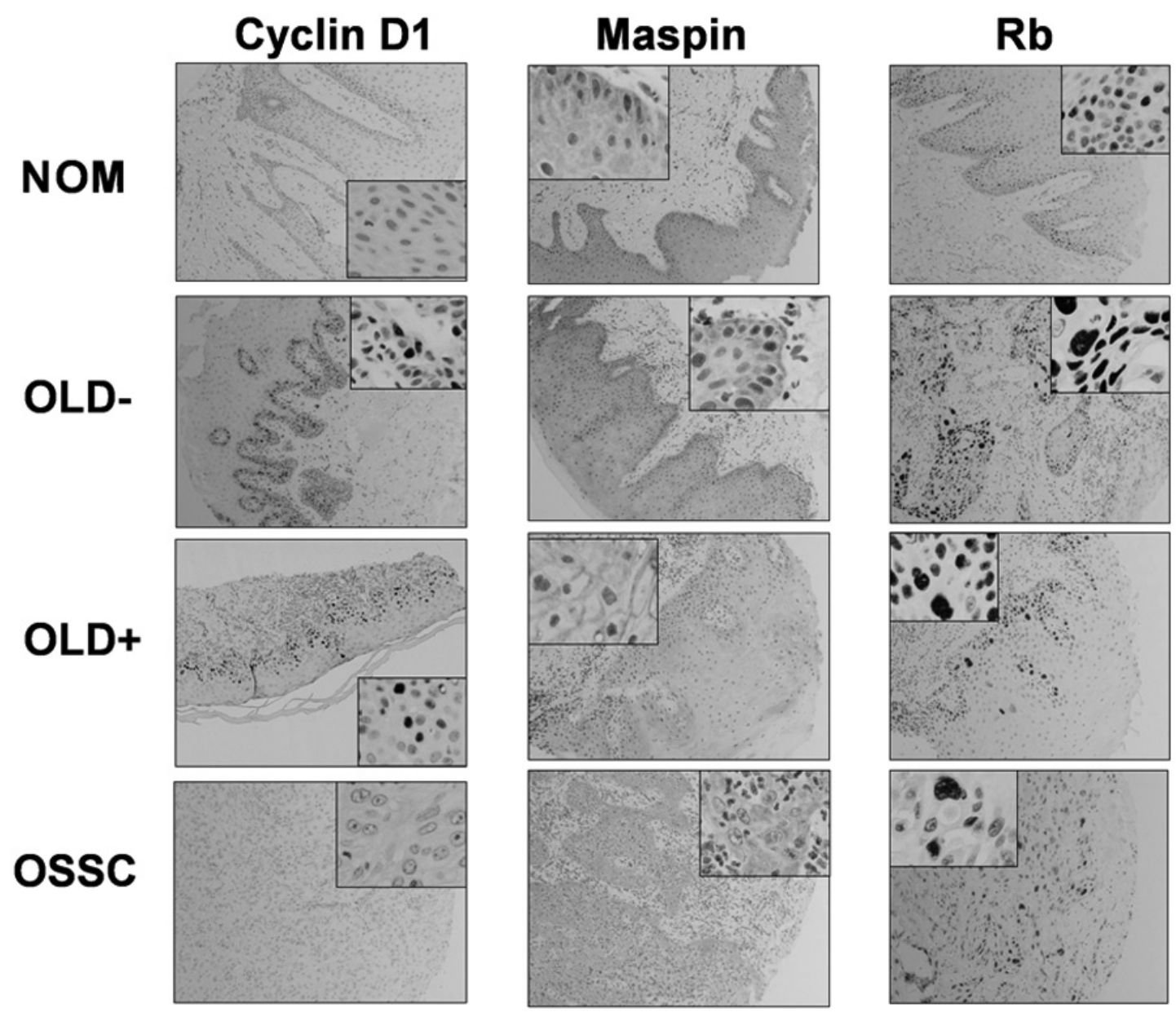

Figure 1. Expression of p16-pRb senescence pathway markers, cyclin D1 and Rb, and the epithelial senescence marker, maspin. NOM, normal oral mucosa; OLD+, leukoplakia with dysplasia; OLD-, leukoplakia without dysplasia; OSCC, oral squamous cell carcinoma.

Expression of the three markers of this pathway was more frequent in OLD+ vs. OSCC samples, but only the increase in maspin was statistically significant $(\mathrm{P}=0.036)$. Cyclin $\mathrm{D} 1$ expression was occurred more frequently in the OLD+ group (54.5\%), whereas maspin and $\mathrm{Rb}$ expression occurred more frequently the OLD- group (100 and 92.8\%, respectively). 
Table III. Expression of ARF-p53 senescence pathway markers.

\begin{tabular}{|c|c|c|c|c|c|c|c|c|c|}
\hline \multirow{2}{*}{$\begin{array}{l}\text { Type of } \\
\text { lesion }\end{array}$} & \multicolumn{3}{|c|}{$\mathrm{p} 53$} & \multicolumn{3}{|c|}{ MDM2 } & \multicolumn{3}{|c|}{ Ki-67 } \\
\hline & Positive & Negative & P-value & Positive & Negative & P-value & Positive & Negative & P-value \\
\hline $\begin{array}{l}\text { NOM } \\
(n=20)\end{array}$ & $2(10 \%)$ & $18(90 \%)$ & $0.006^{\mathrm{a}}$ & $0(0 \%)$ & $20(100 \%)$ & $0.007^{\mathrm{a}}$ & $1(5 \%)$ & $19(95 \%)$ & $<0.001^{\mathrm{a}}$ \\
\hline $\begin{array}{l}\text { OLD- } \\
(\mathrm{n}=14)\end{array}$ & $8(57.1 \%)$ & $6(42.8 \%)$ & $1.000^{\mathrm{b}}$ & $5(35.7 \%)$ & $9(64.3 \%)$ & $0.053^{\mathrm{b}}$ & $10(71.4 \%)$ & $3(21.4 \%)$ & $1.000^{\mathrm{b}}$ \\
\hline $\begin{array}{c}\text { OLD+ } \\
(\mathrm{n}=11)\end{array}$ & $5(45.4 \%)$ & $5(45.4 \%)$ & $0.397^{\mathrm{c}}$ & $0(0 \%)$ & $10(90.9 \%)$ & $0.124^{c}$ & $7(63.6 \%)$ & $4(27.2 \%)$ & $1.000^{c}$ \\
\hline $\begin{array}{l}\text { OSCC } \\
(\mathrm{n}=15)\end{array}$ & $11(73 \%)$ & $4(26.6 \%)$ & $<0.001^{\mathrm{d}}$ & $4(26.6 \%)$ & $11(73 \%)$ & $0.026^{\mathrm{d}}$ & $10(66.6 \%)$ & $5(33.3 \%)$ & $<0.001^{\mathrm{d}}$ \\
\hline
\end{tabular}

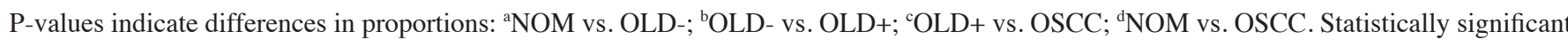
findings are in bold. One sample was excluded (as non-valuable) from p53 staining (1 OLD+); one from MDM2 (1 OLD+) and two from Ki-67 staining (1 OLD- and 1 OLD+). The same OLD+ sample (LC43) was excluded from all staining procedures. MDM2, mouse double minute 2; NOM, normal oral mucosa; OLD+, leukoplakia with dysplasia; OLD-, leukoplakia without dysplasia; OSCC, oral squamous cell carcinoma.
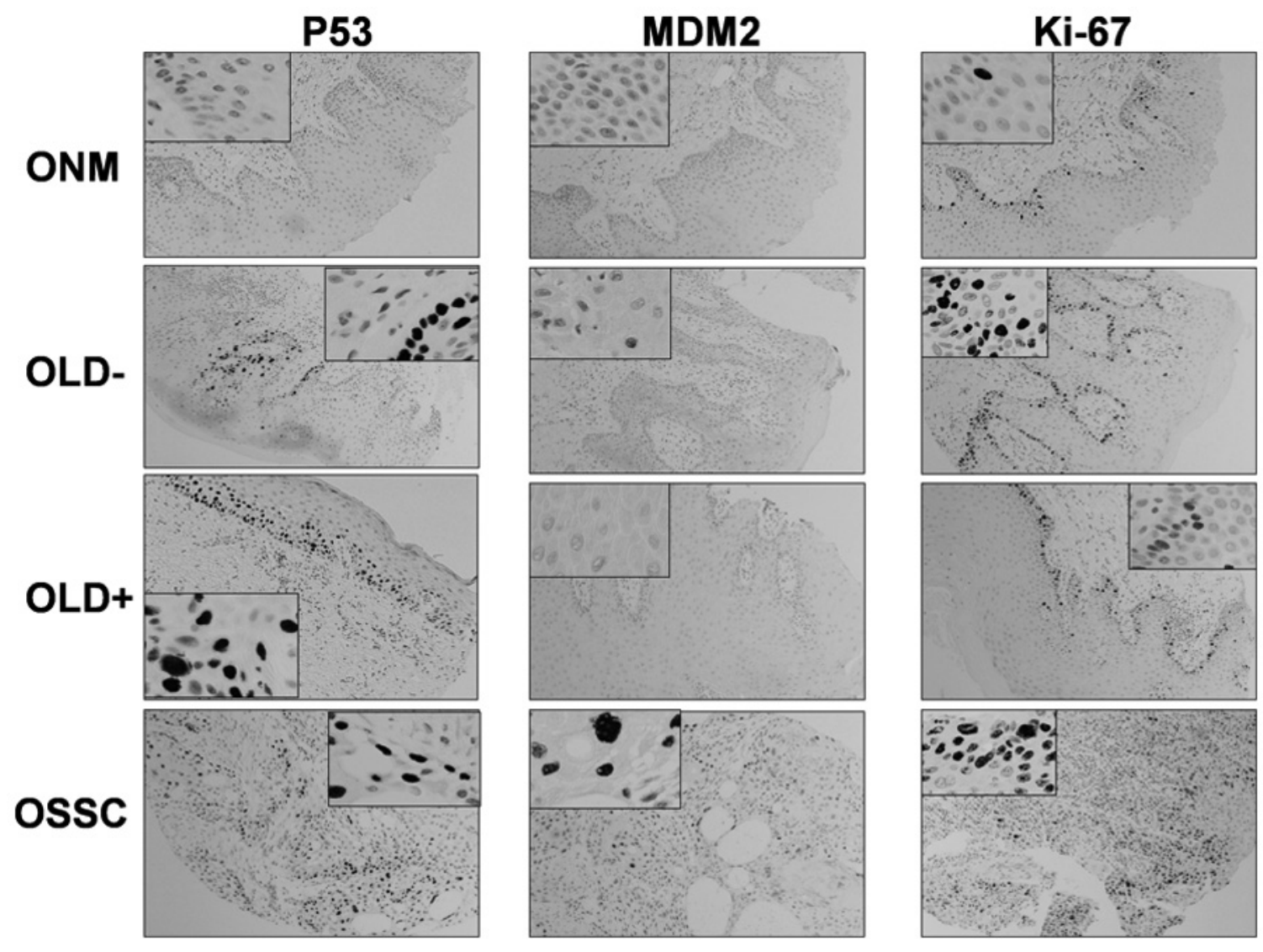

Figure 2. Expression of ARF-p53 senescence pathway markers, MDM2 and p53, and proliferation marker, Ki-67. MDM2, mouse double minute 2; NOM, normal oral mucosa; OLD+, leukoplakia with dysplasia; OLD-, leukoplakia without dysplasia; OSCC, oral squamous cell carcinoma.

ARF-p53 pathway. Fig. 2 shows examples of the expression of markers (p53, MDM2 and cell proliferation marker, Ki-67) of the senescence/apoptosis pathway. Table III shows the immunohistochemistry results (positively and negatively stained samples). The same statistical comparisons were performed as for the p16-pRb pathway. There was a tendency for a more frequent expression of 553 and MDM2 in the OLD- group (57.1 and $35.7 \%$, respectively) and in the OSCC group (73 and $26.6 \%$, respectively), and for a less frequent expression in the OLD+ group (45.4 and $0 \%$, respectively), than that in the NOM group (10 and 0\%, respectively). The expression of these markers was significantly more frequent in the OLD- group 
than in the NOM group, as also observed for the proliferation marker, Ki-67.

\section{Discussion}

Cellular senescence and apoptosis are considered powerful tumour suppressor mechanisms through their control of the proliferative potential of cells (3). Cellular senescence, or permanent arrest of the replicative capacity of cells, has been demonstrated in vitro (1) and in vivo. Animal studies have demonstrated the presence of senescent cells in premalignant epithelial lesions, but not in carcinomas of the same tissues (3,6-8), thus supporting the anti-tumour function of OIS. Various studies have been published on senescence markers in human epithelia, but few at the oral level, and little data are available on precancerous lesions $(9,10)$. The study of premalignant lesions is of particular relevance due to the potential of applying preventive measures.

Analysis of different proteins in the same samples, using TMAs, is valuable to determine whether alterations have a synergistic or cooperative effect on carcinogenesis. Alteration of the p16-pRb pathway is regarded as an early event in dysplasia acquisition and that the involvement of the two pathways, $\mathrm{pRb}$ and p53, is associated with malignant transformation and a poor prognosis in oral carcinoma (10). Our detection of alterations in the two pathways in premalignant lesions indicates that these are early events. Our results also confirm the anti-tumour function of senescence, since the expression of markers of the specific senescence p16-pRb pathway was higher in the precancerous lesions than in the carcinoma ones. Expression findings indicate an increase in the function of these markers in premalignant lesions in comparison to healthy tissue, and a decrease in carcinomas. Our findings for the ARF-p53 pathway also suggest there is not only increased proliferation (Ki-67 expression) during oral carcinogenesis, but also activation of p53- and MDM2-mediated senescence programmes.

Regarding the senescence markers studied, p53 is a widely studied tumour suppressor oncogene (11), whose mutation or loss has been reported in half of all human cancers. In the remaining tumours, p53 maintains its wild-type status, but its function is inhibited by the MDM2 oncoprotein (12). Certain studies have correlated the overexpression of p53 to angiogenesis and tumour recurrence (13). Results from the present study are consistent with this correlation, since p53 expression increased from $45.4 \%$ in OLD+ to $73 \%$ in carcinomas. MDM2 acts as a p53-negative regulator, which maintains p53 at appropriate levels of expression, and as in the case of p53, published findings have been controversial (14). The expression of the polymorphism SNP 309 has been analysed and revealed to increase the transcription, and therefore overexpression of MDM2, leading to the consequent suppression of p53 (14). Few studies are available regarding this polymorphism in oral carcinomas, and not all of these reports have found an association with increased cancer risk $(14,15)$. Modulators of the p16-pRb senescence pathway, cyclin D1 and Rb, control the progression of the cell cycle from $\mathrm{G}$ to $\mathrm{S}$ phase, and alterations in these proteins have been reported in oral epithelial dysplasia lesions (10). The hypophosphorylated state of $\mathrm{pRb}$ is essential for progression of the cell cycle to the $S$ phase. An absence of $\mathrm{pRb}$ expression was found in a high percentage of premalignant oral lesions (64\%) and oral carcinomas (70\%), which is attributed to the loss of the cycle-regulating functions linked to this protein (16). In the present study, we analysed non-phosphorylated $\mathrm{Rb}$ and found, as predicted, lower expression in the cancerous stage, in accordance with other studies (17). Overexpression of cyclin D1 has been reported in oral cancer (18) and even in premalignant lesions (16) as being related to poor prognosis in the majority of studies (18-20). However, a study attempting to explain this overexpression by analysing two polymorphisms, A870G and C1722G (21), did not yield conclusive results.

In the present study, significant differences were only observed for maspin, thus indicating its potential role as a prognostic marker in oral precancer. Maspin is a tumour suppressor, which inhibits tumour-induced angiogenesis and tumour cell motility, invasion and metastasis (22). Studies on maspin have almost exclusively investigated established malignant lesions and have published contradictory results. Certain studies revealed no correlation between protein expression and prognosis (23), while other studies correlated its overexpression with a better $(22,24)$ or worse $(9)$ prognosis of carcinoma lesions. These differences may be in part due to the fact that maspin is primarily expressed in the cytoplasm, but may also be observed in the nucleus, secretory vesicles and cell surface, thus this subcellular partition may affect the function of this protein (22). It is also possible that the role of maspin differs according to the cell type. However, the majority of studies of OSCC $(22,24)$, including the present study, indicate that maspin has a protective role against the oncogenic process, although certain investigators found no correlation (23) or even reached the opposite conclusion (9).

Our observation of a higher frequency of expression of three markers of the p16-pRb pathway (cyclin D1, Rb and maspin) in leukoplakia lesions with dysplasia compared to OSCC lesions appears to corroborate the tumour suppressor role of cell senescence. However, only maspin demonstrated statistically significant differences, supporting its value as a prognostic marker in oral precancer. The expression of markers of the p53-ARF senescence pathway (p53 and MDM2) was significantly higher in leukoplakia than in healthy oral mucosa, suggesting a correlation of these early markers with early events in oral carcinogenesis.

In conclusion, only maspin showed differences in its expression between precancerous and malignant lesions. However, the results of this preliminary cross-sectional investigation should be confirmed and expanded by studies with larger sample sizes that consider a wider range of senescence pathway markers, including P16, P14, P15, P21, DCR2, DEC1 and senescence-associated-beta-galactosidase.

\section{Acknowledgements}

This study was funded by the Fundación Mutua Madrileña, 5251146, Madrid, Spain.

\section{References}

1. Hayflick L and Moorhead PS: The serial cultivation of human diploid cell strains. Exp Cell Res 25: 585-621, 1961.

2. Ohtani N, Mann DJ and Hara E: Cellular senescence: its role in tumor suppression and aging. Cancer Sci 100: 792-797, 2009. 
3. Collado M, Gil J, Efeyan A, Guerra C, Schuhmacher AJ, Barradas $\mathrm{M}$, et al: Tumour biology: senescence in premalignant tumours. Nature 436: 642, 2005.

4. Nickoloff BJ, Lingen MW, Chang BD, Shen M, Swift M, Curry J, et al: Tumor suppressor maspin is up-regulated during keratinocyte senescence, exerting a paracrine antiangiogenic activity. Cancer Res 64: 2956-2961, 2004.

5. Parkin DM, Pisani P and Ferlay J: Global cancer statistics. CA Cancer J Clin 49: 33-64, 1999.

6. Chen Z, Trotman LC, Shaffer D, Lin HK, Dotan ZA, Niki M, et al: Crucial role of p53-dependent cellular senescence in suppression of Pten-deficient tumorigenesis. Nature 436: 725-730, 2005.

7. Michaloglou C, Vredeveld LC, Soengas MS, et al: BRAFE600-associated senescence-like cell cycle arrest of human naevi. Nature 436: 720-724, 2005.

8. Braig M, Lee S, Loddenkemper C, Rudolph C, Peters AH, Schlegelberger B, et al: Oncogene-induced senescence as an initial barrier in lymphoma development. Nature 436: 660-665, 2005.

9. Vered M, Allon I and Dayan D: Maspin, p53, p63, and Ki-67 in epithelial lesions of the tongue: from hyperplasia through dysplasia to carcinoma. J Oral Pathol Med 38: 314-320, 2009.

10. Soni S, Kaur J, Kumar A, Chakravarti N, Mathur M, Bahadur S, et al: Alterations of $\mathrm{Rb}$ pathway components are frequent events in patients with oral epithelial dysplasia and predict clinical outcome in patients with squamous cell carcinoma. Oncology 68: 314-325, 2005

11. Itahana K, Dimri G and Campisi J: Regulation of cellular senescence by p53. Eur J Biochem 268: 2784-2789, 2001.

12. Shangary S, Qin D, McEachern D, Liu M, Miller RS, Qiu S, et al: Temporal activation of $\mathrm{p} 53$ by a specific MDM2 inhibitor is selectively toxic to tumors and leads to complete tumor growth inhibition. Proc Natl Acad Sci USA 105: 3933-3938, 2008.

13. Lavertu P, Adelstein DJ, Myles J and Secic M: P53 and $\mathrm{Ki}-67$ as outcome predictors for advanced squamous cell cancers of the head and neck treated with chemoradiotherapy. Laryngoscope 111: 1878-1892, 2001.

14. Tu HF, Chen HW, Kao SY, Lin SC, Liu CJ and Chang KW: MDM2 SNP 309 and p53 codon 72 polymorphisms are associated with the outcome of oral carcinoma patients receiving postoperative irradiation. Radiother Oncol 87: 243-252, 2008.
15. Huang SF, Chen IH, Liao CT, Wang HM, Liou SH, and Hsieh LL. Combined effects of MDM2 SNP 309 and p53 mutation on oral squamous cell carcinomas associated with areca quid chewing. Oral Oncol 45: 16-22, 2009.

16. Campo-Trapero J, Cano-Sánchez J, Palacios-Sánchez B, Sánchez-Gutiérrez JJ, González-Moles MA and BasconesMartínez A: Update on molecular pathology in oral cancer and precancer. Anticancer Res 28: 1197-1205, 2008.

17. El-Naggar AK, Lai S, Clayman GL,Zhou JH, Tucker SA, Myers J, et al: Expression of p16, Rb, and cyclin D1 gene products in oral and laryngeal squamous carcinoma: biological and clinical implications. Hum Pathol 30: 1013-1018, 1999.

18. Liu HS, Lu HH, Lui MT, Yu EH, Shen W, Chen YP, et al: Detection of copy number amplification of cyclin D1 (CCND1) and cortactin (CTTN) in oral carcinoma and oral brushed samples from areca chewers. Oral Oncol 45: 1032-1036, 2009.

19. Myo K, Uzawa N, Miyamoto R, Sonoda I, Yuki Y and Amagasa T: Cyclin D1 gene numerical aberration is a predictive marker for occult cervical lymph node metastasis in TNM stage I and II squamous cell carcinoma of the oral cavity. Cancer 104: 2709-2716, 2005

20. De Vicente JC, Herrero-Zapatero A, Fresno MF and López-Arranz JS: Expression of cyclin D1 and Ki-67 in squamous cell carcinoma of the oral cavity: clinicopathological and prognostic significance. Oral Oncol 38: 301-308, 2002.

21. Sathyan KM, Nalinakumari KR, Abraham T and Kannan S: CCND1 polymorphisms (A870G and C1722G) modulate its protein expression and survival in oral carcinoma. Oral Oncol 44: 689-697, 2008

22. Marioni G, Gaio E, Giacomelli L, Bertolin A, D'Alessandro E, Stramare R, et al: Maspin subcellular localization and expression in oral cavity squamous cell carcinoma. Eur Arch Otorhinolaryngol 265: S97-104, 2008.

23. Cho JH, Kim HS, Park CS, Kim JK, Jung KY, Shin BK, et al: Maspin expression in early oral tongue cancer and its relation to expression of mutant-type p53 and vascular endothelial growth factor (VEGF). Oral Oncol 43: 272-277, 2007.

24. Yasumatsu R, Nakashima T, Hirakawa N, Kumamoto $Y$, Kuratomi Y, Tomita K, et al: Maspin expression in stage I and II oral tongue squamous cell carcinoma. Head Neck 23: 962-966, 2001. 